\title{
Personal Data Collection in the Workplace: Ethical and Technical Challenges
}

\author{
Judy Bowen, Annika Hinze, Christopher Griffiths, Vimal Kumar, David Bainbridge \\ Computer Science Department \\ University of Waikato, New Zealand \\ \{jbowen,hinze,kumar,bainbridge\}@waikato.ac.nz, cjgg1@students.waikato.ac.nz
}

\begin{abstract}
Forestry is a dangerous work environment and collecting data on site to identify and warn about hazardous situations is challenging. In this paper, we discuss our attempts at creating continuous data-collection methods that are ethical, sustainable and effective. We explore the difficulties in collecting personal and environmental data from workers and their work domain. We also draw attention to the specific challenges in designing for sensor-based, wearable rugged loT solutions. We present a case-study, comprising of a number of experiments, which exemplifies the work we have been undertaking in this domain. The case study is based on our approach to developing a robust, trusted Internet of Things (IOT) solution for dangerous work environments (specifically the forestry environment). We focus the results of this case- study on both the technical successes and challenges as well as the personal and ethical challenges that have been elicited.
\end{abstract}

Personal Data Collection. Hazardous environments. Rugged loT, Ethics. Wearables.

\section{INTRODUCTION}

New Zealand has around 1.8 million hectares of plantation forests and the industry contributes roughly $4 \%$ of national GDP to the economy. Forestry also has the highest fatality and injury rate of any industrial sector in NZ (since 2008 there have been 32 fatalities) and has New Zealand's highest rate of workplace injuries with claims to the NZ accident compensation scheme (ACC) in excess of two million NZ dollars each year. An independent review of all involved in the sector (using interviews and self- reporting) identified potential contributors to the poor safety record Adams et al. (2014). These included a lack of training; worker fatigue; poor health and safety processes. As a result a number of recommendations were made based around initiatives such as increased codes of practice, wider participation in training and certification for workers, the creation of new safety action groups etc. However, there was no deeper consideration of the wider underlying causes nor practical proposals for how to identify and prevent unsafe work practices.

While the specific NZ forestry setting is unique, other outdoor-based and labour-intensive industries such as mining, haulage, all-terrain farming and fishing encounter similarly hazardous situations. Known pressure points are again fatigue, de-hydration, distraction, isolated work, remote locations, inexperienced and poorly paid staff, and time pressures. Our initial interest in this domain was motivated by finding ways to unobtrusively gather large amounts of data from forestry workers in order to generate an actual data set of work and environmental factors (rather then self-reported data) from which to understand the working environment and identify worker fatigue (a known cause of accidents and contributor to risk). There are well-known, and well-studied techniques for using biometric data to indicate and measure fatigue (we discuss these in more detail below) but these are typically laboratory-based and invasive and therefore not suitable for in-situ workplace monitoring. We are interested in finding suitable technological solutions to replicate such measures in an unobtrusive fashion using technology that can be easily deployed in an outdoor setting. Ultimately we would like to use real-time data capture to monitor and understand worker metrics with a view to being able to identify hazardous situations as they arise, and intervene as appropriate. The overarching aim, therefore, is that of reducing the high accident rate in NZ forestry.

There are many challenges inherent in collecting observational data in workplace environments. Human Work Interaction Workshop (HWID) (2015) specifically focused on design for challenging work environments and how to collect relevant data to inform such design. Common themes emerged from

(C) Bowen et al. Published by BCS Learning and 
a variety of different work domains studied, such as safe access to industrial sites, ethical considerations of monitoring employees (including use of, and access to, data) and finding unobtrusive study methods. Our own initial studies, which looked at the use of lightweight and cheap data gathering tools (such as activity trackers) encountered similar problems. Early on in our work it became clear that the technical, ethical and sociological challenges of implementing worker tracking (even on a small scale) required us to find alternative approaches which better addressed these.

Over the course of subsequent experiments it became clear that we needed to measure a variety of different metrics (beyond those offered by basic activity trackers) and develop a better way of understanding the personalised implications of fatigue for workers. This has led to our current approach, which we present in our case study. This seeks to adopt a new style of loT (the Rugged Internet of Things or RloT) as a possible solution. This in turn creates additional challenges, which we also discuss.

This paper therefore addresses two problems. The first is the difficulty in collecting personal and environmental data from workers and their work domain. We address this by proposing a specialised sensor-based loT solution for outdoor environments. The second is the technical and ethical issues that arise from this proposed solution. Our contributions are the insights we provide into the problems associated with data gathering in hazardous work environments and our proposal of how to move forward using a new version of loT (RloT), which is not only suitable for use in rugged and unconnected environments but also considers new mechanisms for data security and privacy. The loT solution we are developing is not only truly body/person-centric, but is also designed to support people's safety on a day-to-day basis.

\section{BACKGROUND TO NZ FORESTRY}

An investigation into the role that rest and recovery play in accidents and injury of workers was undertaken by Lilley et al. (2002). This relied on selfreporting and involved 367 workers responding to a self-administered questionnaire. The results showed that $78 \%$ of workers reported experiencing fatigue at work at least some of the time and the study concluded that the combination of slim margin for error and impairment due to fatigue constituted a significant risk factor within the industry. In an attempt to gain more detailed data, Parker (2010) conducted a study using wearable video cameras to capture forestry worker behaviours. This work was limited by the small number of participants (due to equipment costs) and the time and expertise required to analyse the footage to understand what was being observed. Adams et al. (2014) conducted another study using self-reporting specifically focusing on forestry, with the results outlined in our introduction.

In general, robotic solutions, which are applied elsewhere in forestry, do not work well in the extreme New Zealand terrain, although there is ongoing research in this area to try to adapt equipment or develop new machinery to remove humans from the work environment. Live observation and in-situ monitoring are not suitable in many work conditions and particularly do not work in hazardous work environments. For example, Parker's initial observational studies elicited more about the practice of keeping on-site visitors safe than it did about typical worker behaviours. Some of the specific challenges of data gathering in NZ forestry have been reported in (Bowen et al. 2015b), but here we describe a case study we have carried out in order to identify both the practical requirements as well as the philosophical, ethical and social implications of such work when we endeavour to introduce novel technological solutions into industrial environments.

\section{RELATED WORK}

We focus on related work in three key areas: uses, effects and ethics of monitoring workers; measuring fatigue, activity, recovery and response times; using sensors and loT solutions in work domains.

\subsection{Tracking of Workers}

Employee monitoring and tracking is not a new idea. Different approaches have been used to consider issues such as productivity, health and safety and security since the early days of the factory floorwalker (human observation of worker productivity) and the punch-in time clock used to ensure workers arrived on time and did not leave early. As technology has advanced, so too have the methods used for monitoring and tracking workers. Any form of monitoring of employees can create tension between employers and employees.

Botan (1996) reports on a survey of 465 employees on their attitudes of workplace surveillance. He found that irrespective of the motivations for the surveillance, most workers felt untrusted by their employers and that this was likely to be the first step in other management interventions that would not be in the employee's best interests. Kortuem et al. (2007) discussed the use of worker tracking specifically for Health and Safety purposes. This considered the use of a vibration monitoring technique for industrial workers aimed at reducing a condition called "Vibration White Finger". They considered whether the use of (ubiquitous computing in this instance) could play a role in 
making industrial workplaces safer. They found that even in an example where the monitoring was intended to keep workers safer there was still a perception that such a system could be used to exert control over employees, for example by creating accurate logs of worker activities. Such perceptions existed even when the reality of the monitoring did not include such aims.

The proliferation of personal activity trackers in recent years has given rise to a new type of worker tracking. Firstly there are companies who seek to promote the health of their workers by encouraging them to be active and provide trackers for personal employee use to support this. For example Target in the U.S have offered to give FitBit trackers to all of their workers to increase awareness about healthier lifestyles (Mangan, 2015). In a similar manner, although with more focus on rewarding adherence, oil company BP track step counts of workers and offer lower health care premiums to those who meet certain criteria (Olson, 2015). According to technology research company Gartner, in 2013 about 2,000 companies offered their employees fitness trackers. In 2014 this rose to around 10,000, and companies such as FitBit now have dedicated partnerships with organisations to provide large numbers of trackers ad personalised data provision. The collection of such personal data and its use raises many ethical questions about how such data is used and who has access to the information - for example what happens to the employee who does not meet the fitness criteria defined by their employer? We discuss this later in the paper as it pertains to our case study and our opinions as researchers collecting such data, as well as employee reactions and 'buy in' to such initiatives.

\subsection{Studies into Causes and Effects of Fatigue}

The biometric measures we are proposing to capture, along with their meanings and effects, have been well studied in the field of psychology. Here we primarily focus on the following topics: definitions and effects of fatigue; activity, fatigue and recovery; fatigue and response times; heart-rate variability as an indicator of stress and fatigue.

Fatigue is typically classified into two general types, mental fatigue that affects an individual's cognitive processes and physical fatigue that affects an individual's ability to maintain physical actions. There is some contention over this division though with some researchers believing that fatigue is a single general state that is driven by physiological responses to energy expenditure of whichever category (Hockey and Ebrary 2013). Studies of physical fatigue typically require participants to undertake physically demanding tasks either for a pre-determined period of time or until they are unable to continue. A variety of measurements are compared pre- and post- task to evaluate the effect of the activity and extent of the fatigue. For example Kumar et al. (2004) measured oxygen uptake, ventilation, heart rate, blood oxygenation, blood volume and took electromyographic readings while subjects performed a physically demanding exercise, and reported a steady reduction in force exerted over the duration of the task.

Mental fatigue has been shown to affect task motivation (v. d. Linden et al. 2003) and high levels of mental fatigue have been show to result in a loss of efficiency and lower productivity of workers (Murata et al. 2005). Like physical fatigue, mental fatigue may be the result of fatiguing activities (cognitive processes) but it is also linked to disturbed, or lack of, sleep (Äkerstedt et al. 2002). While physical fatigue can be measured by way of ability to exert force or perform activity (as above), measuring the effects of mental fatigue is less straightforward. One important (for our work) correlation that has been demonstrated is the effect on reaction times of individuals who are fatigued. Galton (1889) developed a simple reaction time (SRT) test which recorded a participant's response to a simple stimulus. This early test still forms the basis for several variations that have been developed to measure SRT and it is also used as the basis for the choice reaction time test (CRT) which records the time it takes a participant to choose a correct response from a number of alternatives. Of particular interest is the evidence showing that reaction time is adversely affected by both physical and mental fatigue (Brisswalter et al. 1997) suggesting we may see slower reaction times in physically demanding jobs, such as those found in forestry.

In addition to fatigue indicators such as SRT and CRT, there are changes in the autonomic nervous system when an individual is under stress (again both physical and mental). One key indicator that can identify this is heart- rate variability (HRV) which is the change in the inter- beat interval of the heart. A higher variability indicates higher levels of stress and corresponding fatigue and has been shown to be caused by work-induced cognitive stress (Chandola et al. 2008) as well as physical activity (Kaur et al. 2014). The increase in wearable technology capable of recording HRV has led to an increase in its use as a stress and fatigue measurement tool for athletes as well as ordinary individuals.

The majority of the studies described above, and many similar or complementary studies, are conducted in controlled environments (typically a laboratory setting) with specialised equipment and involve large-numbers of participants. This enables specific variables to be measured and controlled for required circumstances. For example, simulated driving laboratories can be used to investigate not just the fatiguing effects of driving in general, but 
rather the effects of particular driving conditions over specified periods for large numbers of test subjects (see for example Charlton and Baas (2006); Charlton and Starkey (2013)). Our intention is not try to replicate such studies or re- investigate known results from literature. Rather we want to find out if we can replicate the results of such studies using low-cost and light-weight measurement techniques in real-world settings. If we can do so, then we can rely on such technologies for in-situ monitoring of forestry workers with the confidence that the implications we draw from the measurements are based upon empirical studies conducted in controlled environments.

\subsection{Using Sensors and IOT for Personal Monitoring}

The Internet of Things is predominantly discussed in terms of a self-configuring network connecting objects in 'smart' homes and businesses, with a strong focus on the objects and environments. Typical applications are smart buildings, smart homes, ambient intelligence, and mobile healthcare. These applications assume large-scale and reasonably- stable computation and sensor constellations.

Even IoT applications that use rather fluid sensor constellations typically make these assumptions. For example, participatory sensing or crowd-sensing (Jaimes et al. 2015) are activities that engage the public to place sensors in regions of interest to gain large sample sizes. However, while the sensors, e.g. in the urban surface project (Kuznetsov and Paulos 2010), may be dynamically placed by the public, the urban computing environment itself is wellestablished and stable.

In contrast, our domain has a strong concern with the human body. Communication may be established via a Body Area Network (BAN) in collaboration with a Personal Area Network (PAN). Some smart home applications are treated as an extension of a body area network, e.g., for healthcare applications (Gubbi et al. 2013). Again, most of these can rely on a stable network environment with which sensors can securely communicate. Bodycentric systems use environmental, wearable, and implanted sensors. There are already a number of simple wearable technology applications, such as a T-shirt that visualises air quality monitoring results (Kim et al. 2010) or cycling helmets displaying heartrate data (Walmink et al. 2014). In these cases, the sensing is instantly translated into the visualisation, without recording capability nor any links to the wearer's personal activity context. Some projects use RFID technology and IoT communication for personal health- care applications (Amendola et al. 2014) and for gathering information (temperature, humidity, and other gases) about the user's living environment. For example, Negi et al. (2011) and
Adams et al. (2009) combine sensors with GPS to create a wearable personal air monitors. Wearable systems designed for outdoor use often rely on Bluetooth communication between GPS, sensors and smartphones (Honicky et al. 2008), possibly transferring data to central collection points via GPRS (Dutta et al. 2009). Inside buildings the use of GPS is limited and other indoor positioning systems are employed for location- based monitoring (Brown et al. 2016). Some of the applications focus on realtime monitoring of workers to protect them from environmental hazards, such as overexposure to air pollution (Fathallah et al. 2016).

Many of these health-related applications use loT architectures that are akin to smart-city proposals, which are used to support people with disabilities (e.g., Domingo (2012)). Others use stand-alone body-focused systems, such as the Xbox Kinect. For example, González-Ortega et al. (2014) use 3D computer vision system for cognitive assessment and rehabilitation. These systems assume the support of powerful computing networks, often in a localised setting. Rohokale et al. (2011) proposed using a cooperative loT network for rural healthcare, which is akin to ad-hoc wireless sensor networks in which each node acts as both sensor and relay. This work predominantly focuses on establishing communication with no concern for security or wearability of the equipment.

Scant attention has been paid to the communication and security of data when the loT devices interact autonomously Roman et al. (2013). Secure and trustworthy computing typically focuses on resource-rich environments or hard security measures (e.g., encryption, signatures and certificates) that are typically energy hungry (e.g., Kumar and Madria (2015); Kothmayr et al. (2013)). Only a few studies address the specific challenges of trust in loT (e.g., Bao and Chen (2012); Lacuesta et al. (2012)), and none take into consideration the characteristics of rugged environments, making their findings not applicable in such environments. Trust in loT data collection at user level has recently received more public attention, with users becoming aware of the potential for mis-use of information collected (Brennan 2015). Limiting collection of data is difficult when safety requires data collection and devices record very personal data via sensors embedded in clothing or even implanted.

Interaction design in the loT space makes it tempting to merely or overly focus on the objects - the 'things' in the Internet of Things (Jenkins 2015). Our problem domain has two interaction aspects: sensing of data and feedback to workers. In this paper, we consider the challenges of sensing, with a strong focus on the interplay between the objects and the humans involved.

\section{THE RUGGED INTERNET OF THINGS (RIOT)}


We believe that the new generation of lightweight, wearable technology and sensors of the Internet of Things (loT) can help in identifying hazardous situations in work environments such as forestry, ultimately preventing fatalities. There are, however, many challenges in doing so. The use of loT has already been embraced in some hazardous work environments, such as mining (Pye 2015). However most of these projects focus on specific environments where infrastructure is not an issue. Such ideas are built on the assumption of the continuous availability of computational power (in the form of cloud computing), high bandwidth (in the form of WiFi and cellular networks) and energy, since devices can be plugged in.

Many of those assumptions do not hold in rural, agricultural and forestry settings. Resources in these places may only be available intermittently. Such environments are characterised by lack of available bandwidth, computation constraints, energy constraints, and very importantly limited interaction between devices. Existing loT technologies, which rely on the aforementioned assumptions, cannot cope in such rugged environments. For loT to work successfully and safely in rugged environments we must recognise that the standard assumptions do not work and provide alternatives. Our current experimental setup mixes fixed access points with temporary storage solutions (mobile phones) to ensure sensor data is not lost as workers move in and out of connectivity.

We therefore set out to explore the following three aspects:

Relevant data: What sort of data might be relevant in order to determine worker fatigue and unsafe situations?

Suitable Collection: Considering the data we might wish to collect, what are appropriate and effective ways of collecting such required data from forestry workers?

Analysis \& use How can we analyse and use the obtained data (online or off-line) such that it would be of use in ensuring safety in dangerous work environments?

We present a summary of our analysis of relevant data to be collected in the next section. We then present in detail a case study that looks into suitable methods for collecting data in the forestry environment. Explorations of online use of the data have already begun and are also part of our future work.

\section{RELEVANT DATA: ANALYSIS}

As discussed above, our RloT targets the problem of a 'smart landscape' in which disconnectedness and harsh operating conditions are the norm.
Business and personal data are highly sensitive and possible interference through attempted data access or malicious data inserts have to be prevented. In addition, as it is known that workers may be suspicious about how any of the collected data is used, even if they agree to participate they may not comply. Therefore, spurious or suspicious data may be the result of either malicious interference from external entities or due to worker disruption.

Data we wish to collect can be categorised across several different dimensions. First, we consider the privacy considerations for the data and whether it should be considered as:

- personal $[P]$ - only the owner should have access to the unconsolidated and unanonymised data, identification should only be possible in specified emergency scenarios

- business-sensitive $[\mathrm{B}]$ - needs to be concealed from external entities as it may reveal properties of the work environment that can be considered commercially sensitive

Secondly we consider the requirement for when data should be available for collection (frequency and availability) which can also be divided into two groups:

- Continuous $[\mathrm{C}]$ - where it is essential that data is collected in an uninterrupted manner

- Infrequent [I] - data may be provided at varying intervals throughout the day

Table 1 gives an overview of the proposed data to be collected and its categorisations. These data categories were developed based on prior work with high- performance athletes Tavares et al. (2016) and industry engagement with forestry workers Bowen et al. (2015a,b); Griffiths (2016), as well as analysis of relevant literature.

\begin{tabular}{|l|l|l|l|l|}
\hline Data & $P$ & $B$ & $C$ & $I$ \\
\hline Activity & $X$ & & & $X$ \\
Ambient temperature & & & & $X$ \\
Breathing & $X$ & $X$ & $X$ & \\
Calorific burn & $X$ & & & $X$ \\
Core body temperature & $X$ & $X$ & & $X$ \\
Heart-rate variability & $X$ & $X$ & $X$ & \\
Hill climbing & $X$ & & & $X$ \\
Location & & & $X$ & \\
Reaction times & $X$ & $X$ & & $X$ \\
Sleep data & $X$ & $X$ & & $X$ \\
Vibration & $X$ & $X$ & $X$ & \\
\hline
\end{tabular}

Table 1: Data Categories

\section{SUITABLE COLLECTION: CASE STUDY}

In order to explore suitable data collection methods for worker-related fatigue data in the outdoor forestry 
Table 2: Case Study Phases (data on external participants + forestry workers)

\begin{tabular}{|c|c|c|c|}
\hline Phase & Description & Length & \#Participants \\
\hline$I$ & Activity, steps and sleep patterns & $16+5$ weeks & $4+2$ \\
\hline II & $\begin{array}{l}\text { Activity, steps and response times } \\
\text { Heart-rate variation, physical and mental fatigue activities }\end{array}$ & $\begin{array}{l}3+3.5 \text { weeks } \\
\text { ongoing }\end{array}$ & $1+15$ \\
\hline
\end{tabular}

environment, we designed a case study that comprises three phases (see Table 2). The table provides an overview of collected data for each phase, participants and length of the study. So far, we have conducted Phases I and II of our case study, and are currently undertaking Phase III. We here first describe the case study setup and report on results of Phases I and II; it is too early to report results on Phase III - it is shown here only for completeness. We then discuss our observations, the challenges encountered and lessons learnt from the case study.

We begin by summarising each of the phases wrt. their goals, aims and participants. We then discuss the challenges and problems that were identified by the studies and how they relate to our initial problem statement. We conclude this section with a discussion of the insights obtained from our results and how they contribute to our proposed solution.

\subsection{Case Study Methodology}

We started by looking at how we might predict hazards by harnessing the power of a new generation of lightweight, wearable technology (such as activity trackers). We subsequently investigated different types of sensors (and wearable sensors such as those found within the LifeBEAM Smart Hat (for example) within an loT.

Phase 1. This phase started with a number of experiments performed by the research team over varying periods of time to investigate the properties of activity tracker usage.

The aim of this initial research was to discover any usability issues that might occur when using activity trackers for long-term studies (so requiring them to be worn 24/7) as well as investigating the effects of domain-specific activities (such as operating a chain-saw, driving long distances on bumpy roads, walking in forestry environment etc.) Three researchers wore devices (sometimes more than one at a time for comparison purposes) to track both daily activity and sleep for periods of 6-14 weeks. In addition one researcher kept a diary throughout the same period to enable consideration of particular data points. We then replicated this data collection with two forestry workers over a five week period. The participants were asked to wear an activity tracker (each had a different brand) 24 hours a day, initially for a period of two weeks, and then subsequently for another two weeks, then for one final week. The extensions were due to ongoing problems with the participants use of the trackers (discussed shortly).

Phase 2. The second phase began by one of the researchers undergoing a period of self-monitoring on activity and fatigue levels. Activity was measured based on step- counting, heart-rate and calorific burn (using a Fitbit HR), sleep was measured in terms of quantity and quality (again using the Fitbit $H R$ ) and the effects of fatigue were measured based on reaction times. Reaction time testing used two methods, simple-reaction time testing (SRT) and choice-reaction time testing (CRT). For this study, reaction time was measured using the 'Reaction Time' application designed to measure the time taken to respond to visual stimulus (colour change) and screen touch (response). These experiments ran for a three week period and encompassed activities undertaken by a researcher, covering a mixture of workplace and study activity.

Again we then moved our data collection to in-situ forestry workers. This involved the collection of physiological data by means of a Fitbit Charge HR wrist worn monitoring device and testing of SRT and CRT. Simple and choice reaction time measurements were undertaken at commencement of the participants' work period, during their break time and on completion of the participants' work day. The Deary-Liewald Reaction Time Task application developed by the Centre for Cognitive Ageing and Cognitive Epidemology at the University of Edinburgh Deary et al. (2011) was used for this purpose. SRT testing was completed first with each participant undertaking 15 individual tests. CRT testing was performed secondly with participants undertaking 20 individual tests. Participants were selected from three work crews based at three separate locations (all members of a crew were included where possible) who were each monitored for two periods of 3-5 days. In total there were fifteen participants who were all male, with ages between 17 and 62. Five participants were loader operators, three worked in quality control, three were manual tree fellers, two were process operators and two were log makers.

Crew 1 was a fully mechanised crew with most operations being carried out using plant and machinery. Crew 2 was a primarily manual crew with most operations being performed by workers on the ground (using chainsaws etc.) Crew 3 was a hauler crew who operate in steep terrain using cabling techniques to drag felled trees to the skid site for processing. The working day for all three crews 
(apart from loader operators who typically started the day 2 hours earlier and then broke for the morning meeting) starts with a meeting where tasks are assigned. Directly after this meeting we issued each participant with a Fitbit HR to wear for the day and performed the first of the reaction time tests. Work then commences for around four hours, at which point a 45 minute break occurs when we performed the second reaction time test. Work then re- commences until the end of the day when we performed the final reaction time test and collected the activity trackers for data synchronising and charging.

\subsection{Summary of Results}

We here summarise the case study results wrt. the data quality and suitability of the data collection method.

Phase 1. While the full results from Phase I were reported in Bowen et al. (2015a), here we discuss the key findings from both phases and show how they relate to the aims of the work described. Our experiences of Phase I showed that context-free data can be misleading (high activity levels may not be due to steps but other actions such as driving or even drinking). There were large variances in sleeptracking accuracy (when compared with diary reports as well as measured against state of the art devices like the Readiband) and devices can get in the way of some activities (uncomfortable when typing on a keyboard for long periods or irritating to the skin overnight). This suggests that choice of technology needs to be based on a number of factors and that data needs to be correlated with other variables in order to obtain a clearer picture of its meaning. While different types of tracker reported different values for activity tracking the differences remained consistent (so trends seemed accurate) which means that factors such as comfort and utility in the environment can be used as the dominant choice factor.

The most significant finding from the forestry worker engagement in Phase $I$ is the level of technical difficulties that occurred-participants could not change the mode of the devices to track sleep; devices were lost; mode changes occurred frequently so that data collection was compromised; participants never charged the devices; connectors for uploading data were lost. Of course, it is possible (and indeed likely) that not all of these problems were actually technical but that there were also elements of resistance from the participants to being monitored in this fashion. As we have discussed earlier, in some sense this is not surprising, the monitoring of workers during their private time is potentially controversial. Even though our participants were volunteers and keen to take part, during the initial meeting to set up the study it was clear that there were reservations about some aspects of the monitoring (particularly the sleep monitoring) and what could be identified from the data).

Workers were assured that only anonymous and aggregated data would be available to their boss; however, the fact that their boss might be able to see any data caused considerable concern and might have been the reason for the subtle disruptions and signs of non-compliance we observed. A similar observation was made by Kortuem et al. (2007), as they explored organisational issues of industrial health and safety monitoring system. They had also observed "both a perceived lack of trust and a lack of effective two-way communication between management and operatives".

Phase 2. As the first part of Phase II involved selfmonitoring, and because we had already learnt some of the lessons relating to equipment choice from Phase I, there were overall fewer issues. Our focus for Phase 2 was on identifying correlations between activity and reaction times. Specifically we wanted to see if the data we could collect would correlate with known properties of activity and fatigue (as discussed earlier). Speed of mental processing (SMP) is a means to aggregate data from simple and choice reaction time (SMP $=$ CRT SRT ). A summary of the collected data for Crew 3 is shown in Table 3 . We found that the effect of activity on reaction time varied between participants. SRT showed no common pattern other than a tendency towards being slightly slower at the end of the day than at the start of the day but between those points there was no consistent effect. CRT similarly had no common pattern. The data is personalised but may also depend on role types. For example, the loader drivers $3 E$ and $3 D$ both show improved mental processing times as well as CRT and SRT throughout the day. People were typically found to be consistent within each day but very different from each other. We also observed that not only physical exhaustion may contribute to fatigue but also mental activity.

Table 3: Crew 3 Mean reaction times in msec

\begin{tabular}{|l|l|l|l|l|l|}
\hline Period & Participant & Role & CRT & SRT & SMP \\
\hline Start & $3 A$ & Quality Control & 662 & 701 & -39 \\
\hline Break & & & 649 & 382 & 267 \\
\hline End & & & 525 & 390 & 135 \\
\hline Start & $3 B$ & Quality Control & 624 & 445 & 179 \\
\hline Break & & & 544 & 363 & 181 \\
\hline End & & & 534 & 396 & 148 \\
\hline Start & $3 C$ & Manual Feller & 566 & 392 & 174 \\
\hline Break & & & 566 & 386 & 180 \\
\hline End & & & 569 & 357 & 212 \\
\hline Start & $3 D$ & Loader Operator & 520 & 311 & 209 \\
\hline Break & & & 456 & 321 & 135 \\
\hline End & & & 458 & 277 & 181 \\
\hline Start & $3 E$ & Loader Operator & 1067 & 1016 & 51 \\
\hline Break & & & 820 & 569 & 251 \\
\hline End & & & 742 & 424 & 318 \\
\hline
\end{tabular}


Implications for data collection As a result of Phase I we made a decision that we would not continue with sleep- tracking of the workers; instead it was decided to focus on measuring the effects that fatigue (based on activity) might have. It may be that including some type of self- reporting question regarding sleep quality at the start of the electronic reaction time test may be useful, although we should be mindful that the same ethical issues that could lead to non-compliance with the sleep monitoring may similarly affect the answers given. If workers believe they may be penalised (e.g. sent home as unfit to work) if they select an answer indicating they have slept poorly several nights in a row then they may be reluctant to provide such an answer.

The need for the researcher to be on site several times a day to facilitate the testing also meant that they were able to observe some aspects of the environment which proved to be informative, despite not being part of the overall study plan. The effect of temperature appears to have a bigger impact on reaction time (both SRT and CRT) than activity alone. The biggest effect was seen at temperatures less than $4^{\circ} \mathrm{C}$ with the mean differential across all participants between $2^{\circ} \mathrm{C}$ and $4^{\circ} \mathrm{C}$ on CRT being over 100 milliseconds and on SRT being around 50 milliseconds. However, this needs to be considered in conjunction with the activity data as typically the coldest part of the day is when work commences and as the temperature rises the amount of activity having been performed also increases.

The remote working conditions for each of the forestry crews and lack of facilities contributed to the effect of ambient temperature as an important variable. Work sites are at remote locations with the only welfare facilities available to the crew being the vehicles they travel to work in. There are no fresh water or toilet facilities at any of the locations. Crew 1 had access to a metal shipping container that is used as both the site office and lunch room. This container travels with the crew from production site to production site as the crew moves around. This has an effect on workers' choice of hydration and food throughout the day. In addition the lack of a comfortable, warm, area to take breaks in, and no running water or power meant that breaks were taken as required in a perfunctory manner, rather than being used as an opportunity for workers to relax, make tea/coffee, heat up food, socialise etc. as might be seen in indoor working environments. Power is not supplied to the container and as such no heating is available and crews protect themselves from adverse temperatures by use of clothing layers. Machine operators who spend large parts of the day sitting in unheated machine cabs are particularly affected by cold temperatures, whereas the workers on the ground report finding the summer months where they are unprotected from the heat more physically challenging. Our results also indicated that mental fatigue (again seen by machine operators) appears to have a stronger effect than that of physical activity. It is clear, therefore, that all of these need to be carefully measured and considered in larger scale datagathering activities.

Finally the individual nature of the results seen (particularly the differences in reaction time across workers) indicates that there can be no overall general benchmark applied to determine whether or not a worker is fatigued or has reduced reaction time. Rather we need individual data collected over time to act as a personal benchmark, so deviation from an individual's normal pattern of data is what is important. Again this emphasises the need to build personal data histories as a mechanism for predicting future behaviours. The requirements for collecting, storing and analysing this type of personal data, as well as incorporating environmental and business data leads to a number of further considerations that we discuss next.

\section{DISCUSSION}

The personal stories we uncovered and the insights gathered while doing these studies suggest that the design considerations are not those we traditionally prioritise. Any system used for monitoring workers, even when their safety is our primary concern, must be focussed on their privacy as much as anything else. It may be that we will not create the most effective loT solution or will not include the most optimal data inputs but rather we aim to find the most ethical, robust and secure solution that can do the required job. There are many philosophical, ethical \& social implications of collecting and using this data. Workers already do hard jobs for minimum wage, if we collect data that deems them not fit to work then what happens? There are two distinct parts to our focus, the first is that any data collected is shared (or hidden) appropriately. The second is that workers are kept safe and well.

In Phase I, one of the workers was off sick for a day and at the same time problems were encountered with his monitoring device. This again suggests that there is a fine line between what is acceptable and what is not when it comes to such personal monitoring. It is also clear that it may not always be obvious where a problem lies and if a technology error really is that or if something else is at play. As computer scientists it is tempting to focus on the things we can do rather than the things we should do. If one could market such monitoring solutions as being beneficial to workers, or the elderly or the disabled (as we see with many similar monitoring approach rationales) then we may stray into dangerous areas. Risk assessment for poorly paid workers in dangerous environments is clearly a good 
thing, but if the data is used to send home, or lay off, workers who do not meet the new risk criteria then we must consider the responsibilities we have in this. This is especially true when it is not as simple as saying "worker $X$ is fatigued and will cause a serious accident if he stays at work"; the interplay of various factors is much more subtle than that. While high-performance athletes seem to accept the "labrat" lifestyle where all aspects of their performance may be monitored both in and out of work this comes with benefits for them which allow them to improve and attain higher standards. However most research in this area focusses on the different metrics or studies that can be used to fine- tune athletic performance rather than consider the effect this has on those being studied-particularly when underperformance or lack of adherence to training and nutrition schedules is suggested.

Similarly the choice of the components we include in our RloT solution must be carefully considered. Offthe-shelf sensors and tracking equipment is appealing because of its availability and low cost (easy to deploy quickly to large numbers of people), but much of this is not designed to be secure or private. We have been experimenting with a hat that includes a built-in heart rate monitor which just broadcasts its data via Bluetooth continually and which can potentially be captured by anyone or anything in close enough proximity. Developers of such artefacts for personal use are not typically concerned with such data leakage which may not seem concerning when geared towards lifestyle and fitness. Even presuming we do collect data in a secure and private manner (see Table 1), we still need to ask the questions about who then has access to this data (the workers themselves, their bosses, health and safety bodies etc.) and how it is presented and used. We must be clear about our proposed use of this data and ensure that it cannot be accessed and used for other purposes (performance management of employees for example). This again requires us to treat the data in ways that may not necessarily be the most optimal in terms of the technological solution but which ensures the ethical dimension is acknowledged.

\section{SUMMARY AND FUTURE WORK}

In this paper we have discussed our attempts at creating continuous data-collection methods that are ethical sustainable and effective. We explore the difficulties in collecting personal and environmental data from workers and their work domain and discuss both the technical and ethical issues that arise. We have also presented a case-study that explores data collection methods as part of a robust, trusted Internet of Things (IOT) solution for dangerous work environments. We described considerations for relevant data and suitable collection methods, while exploring the use of lightweight sensors to monitor worker activity levels and response times as fatigue indicators.

As we discussed earlier, the nature of the data we wish to observe and gather includes highly sensitive personal data as well as business-sensitive information. This naturally requires safe methods for storage and communication among trusted partners. However, the very nature of the rugged environments we are working in means that connected devices may be transient due to power limitations, movement in and out of connectivity etc. This means that security and trust must be dealt with dynamically, and the loT includes small lightweight sensors that do not have the capacity for on-board security. Not only does this add a layer of overhead that does not exist in typical loT in terms of management of connectivity, it also means that trust cannot develop over time as in established loT networks. Another element of our research, therefore, is in developing a trust model that can support this type of dynamic connectivity and can react accordingly if devices disappear or newly enter. The model should also be able to differentiate between anomalous and maliciously inserted false data. Reliance on redundancy to partially solve this problem is not necessarily suitable in a rugged environment where the infrastructure to support just the minimum required connectivity is already challenging.

We propose to protect the data through the use of a trust management system. Typically trust amongst IoT components is either confirmed by third parties (which are not available in our setting) or is developed over time (which is not suitable in our dynamic outdoor environment). We use data aggregation and composition to derive valuable safety-related information from the collected sensor data. Our trust model will analyse the data from neighbouring nodes in the loT and classify the data as acceptable or malicious. We started with wearable technologies as a proof-of-concept for our data collection but want to go beyond just collecting data so propose to develop a sensor- based IoT which also provides feedback to workers via wearable technology such as a smart vest.

The next stage for our work is completing Phase III of our case study in which we experiment with monitoring heart-rate variability data and recognition of mental and physical fatigue activities. This will be incorporated into sensors that will be part of our RloT network setup. This will then be employed in field tests to see how well the RloT network performs and to identify potential areas of weakness in the security aspects. We can then move on to analysing the data itself and consider how we will use them insitu in useful ways to help reduce risk. 


\section{REFERENCES}

Adams, C., Riggs, P., and Volckens, J. (2009). Development of a method for personal, spatiotemporal exposure assessment. Journal of Environmental Monitoring, 11(7):1331-1339.

Adams, G., Armstrong, H., and Cosman, M. (2014). Independent forestry safety review - an agenda for change in the forestry industry.

Äkerstedt, T., Fredlund, P., Gillberg, M., and Jansson, B. (2002). Work load and work hours in relation to disturbed sleep and fatigue in a large representative sample. Journal of Psychosomatic Research, 53(1):585 - 588.

Amendola, S., Lodato, R., Manzari, S., Occhiuzzi, C., and Marrocco, G. (2014). RFID technology for iot-based personal healthcare in smart spaces. IEEE Internet of things journal, 1(2):144-152.

Bao, F. and Chen, I. (2012). Dynamic trust management for internet of things applications. In Workshop on Self-aware Internet of things, pages 1-6. ACM.

Botan, C. (1996). Communication work and electronic surveillance: A model for predicting panoptic effects. Communication Monographs, 63:294-313.

Bowen, J., Hinze, A., Cunningham, S., and Parker, R. (2015a). Evaluating low-cost activity trackers for use in large-scale data gathering of forestry workers. In Australian SIGCHI Conference (OZCHI), pages 474-482. ACM.

Bowen, J., Hinze, A., and Cunningham, S. J. (2015b). Into the woods. In INTERACT, pages 137-143. Springer.

Brennan, J. (2015). 5Ps of the Internet of Things: A user-centric approach. Keynote presentation at the European Workshop for Trust \& Identity.

Brisswalter, J., Arcelin, R., Audiffren, M., and Delignieres, D. (1997). Influence of physical exercise on simple reaction time: effect of physical fitness. Perceptual and Motor Skills, 85:1019-1027.

Brown, K., Shaw, P., Mead, K., Kovein, R., Voorhees, R., and Brandes, A. (2016). Development of the chemical exposure monitor with indoor positioning for workplace VOC surveys.

J. of occupational and environmental hygiene, 13(6):401-412.

Chandola, T., Britton, A., Brunner, E., Hemingway, H., Malik, M., Kumari, M., Badrick, E., Kivimaki, M., and Marmot, M. (2008). Work stress and coronary heart disease: what are the mechanisms? European Heart Journal, 29(5):640.
Charlton, S. and Baas, P. H. (2006). Fatigue, workrest cycles, and psychomotor performance of New Zealand truck drivers. New Zealand Journal of Psychology, 30(1):32 - 39.

Charlton, S. G. and Starkey, N. J. (2013). Driving on familiar roads: Automaticity and inattention blindness. Traffic Psychology and Behaviour, 19:121 - 133 .

Deary, I. J., Liewald, D., and Nissan, J. (2011). A free, easy- to-use, computer-based simple and four-choice reaction time programme: The DearyLiewald reaction time task. Behavior Research Methods, 43(1).

Domingo, M. (2012). An overview of the Internet of Things for people with disabilities. Journal of Network and Computer Applications, 35(2):584596.

Dutta, P., Aoki, P., Kumar, N., Mainwaring, A., Myers, C., Willett, W., and Woodruff, A. (2009). Common sense: participatory urban sensing using a network of handheld air quality monitors. In Embedded networked sensor systems, pages 349-350.

Fathallah, H., Lecuire, V., Rondeau, E., et al. (2016). An iot-based scheme for real time indoor personal exposure assessment. In Consumer Communications \& Networking Conference (CCNC), pages 323-324. IEEE.

Galton, F. (1889). An instrument for measuring reaction time. Report of the British Association for the Advancement of Science, 59:784-785.

González-Ortega, D., Díaz-Pernas, F., MartínezZarzuela, M., and Antón-Rodríguez, M. (2014). A kinect-based system for cognitive rehabilitation exercises monitoring. Computer methods and programs in biomedicine, 113(2):620-631.

Griffiths, C. (2016). Investigating tools and methods for data capture of forestry workers. Master's thesis, University of Waikato.

Gubbi, J., Buyya, R., Marusic, S., and Palaniswami, M. (2013). Internet of Things (IoT): A vision, architectural elements, and future directions. Future Generation Computer Systems, 29(7):1645-1660.

Hockey, R. and Ebrary, I. (2013). The psychology of fatigue: Work, effort and control. Cambridge University Press.

Honicky, R., Brewer, E., Paulos, E., and White, R. (2008). N-smarts: networked suite of mobile atmospheric real-time sensors. In Networked systems for developing regions, pages 25-30.

Human Work Interaction Workshop (HWID) (2015). position papers. available online at https://projects.hci. sbg.ac.at/hwid2015/positionpapers. 
Jaimes, L., Vergara-Laurens, I., and Raij, A. (2015). A survey of incentive techniques for mobile crowd sensing. IEEE Internet of Things Journal, 2(5):370-380.

Jenkins, T. (2015). Designing the things of the iot. In ACM Tangible, Embedded, and Embodied Interaction, pages 449-

452. ACM.

Kaur, S., Bhalla, B., Bajaj, S. K., Sanyal, S., and Babbar, R. (2014). Effect of physical and mental stress on heart rate variability in type-A and typeB personalities. Indian Journal of Applied Basic Medical Sciences, 15(20):59-70.

Kim, S., Paulos, E., and Gross, M. (2010). WearAir: expressive t-shirts for air quality sensing. In ACM Tangible, embedded, and embodied interaction, pages 295-296. ACM.

Kortuem, G., Alford, D., Ball, L., Busby, J., Davies, N., Efstratiou, C., Finney, J., White, M. I., and Kinder, K. (2007). Sensor Networks or Smart Artifacts?, pages 465-482.

Kothmayr, T., Schmitt, C., Hu, W., Brünig, M., and Carle, G. (2013). DTLS based security and twoway authentication for the Internet of Things. Ad Hoc Networks, 11(8):2710-2723.

Kumar, S., Amell, T., Narayan, Y., and Prasad, N. (2004). Measurement of localized muscle fatigue in biceps brachii using objective and subjective measures. Muscle Strength, pages 105-121.

Kumar, V. and Madria, S. (2015). Distributed attribute based access control of aggregated data in sensor clouds. In Reliable Distributed Systems (SRDS), 2015 IEEE 34th Symposium on, pages 218-227. IEEE.

Kuznetsov, S. and Paulos, E. (2010). Participatory sensing in public spaces: activating urban surfaces with sensor probes. In ACM Conference on Designing Interactive Systems, pages 21-30. ACM.

Lacuesta, R., Palacios-Navarro, G., Cetina, C., Peñalver, L., and Lloret, J. (2012). Internet of things: where to be is to trust. EURASIP Journal on Wireless Communications and Networking, 2012(1):1-16.

LifeBEAM SmartHat (2017) http://lifebeam.com/shop/smart-hat/

Lilley, R., Feyer, A., Kirk, P., and Gander, P. (2002). A survey of forest workers in New Zealand. Do hours of work, rest, and recovery play a role in accidents and injury? Journal of Safety Research, 33(1):53-71.

Mangan, D. (2016). Target's Fitbit offer to workers may miss its mark. CNBC. http://www.cnbc.com/2015/09/16/targets-fitbitoffer-to-workers-may-miss-its-mark. html

Murata, A., Uetake, A., and Takasawa, Y. (2005). Evaluation of mental fatigue using feature parameter extracted from event-related potential. International journal of industrial ergonomics, 35(8):761-770.

Negi, I., Tsow, F., and Tanwar, K. (2011). Novel monitor paradigm for real-time exposure assessment. Journal of Exposure Science and Environmental Epidemiology, 21(4):419-426.

Olson, P. (2015). Fitbit On Track To Sell Thousands More Devices Through Barclays, GoDaddy And Other Employers. Forbes. http://www.forbes.com/sites/parmyolson/2015/10 /20/fitbit-employers-barclays-godaddywellness/\#6170061b3baa

Parker, R. (2010). Technological advances in the analysis of work in dangerous environments: Tree felling and rural fire fighting. PhD thesis, Massey University, Palmerston North, New Zealand.

Pye, A. (2015). Mining companies embrace the loT. Engineering and Technology Magazine, 10(5).

Reaction Time app available from Google Store https://play.google.com/store/apps/details?id=co m.chingy 1788 .reactiontime

Readiband Sleep Tracker (2017) Fatigue Science. https://www.fatiguescience.com/applications/res earch/

Rohokale, V., Prasad, N., and Prasad, R. (2011). A cooperative loT for rural healthcare monitoring and control. In Wireless Communication, Vehicular Technology, Information Theory and Aerospace \& Electronic Systems Technology, pages 1-6.

Roman, R., Zhou, J., and Lopez, J. (2013). On the features and challenges of security and privacy in distributed Internet of Things. Computer Networks, 57(10):2266-2279.

Tavares, F., Healey, P., Smith, T. B., and Driller, M. W. (2016). Effect of training load on acute fatigue and wellness during an in-season noncompetitive week in elite rugby athletes. In Sport \& Exercise Science New Zealand Annual Conference.

v. d. Linden, D., Frese, M., and Meijman, T. (2003). Mental fatigue the control of cognitive processes. Acta Psychologica, 113(1):45-65.

Walmink, W., Wilde, D., and Mueller, F. (2014). Displaying heart rate data on a bicycle helmet to support social exertion experiences. In ACM Tangible, Embedded and Embodied Interaction, pages $97-104$. 